\title{
Mashup Based Content Search Engine for Mobile Devices
}

\author{
Kohei Arai ${ }^{1}$ \\ Graduate School of Science and Engineering \\ Saga University \\ Saga City, Japan
}

\begin{abstract}
Mashup based content search engine for mobile devices is proposed. Example of the proposed search engine is implemented with Yahoo!JAPAN Web SearchAPI, Yahoo!JAPAN Image searchAPI, YouTube Data API, and Amazon Product Advertising API. The retrieved results are also merged and linked each other. Therefore, the different types of contents can be referred once an e-learning content is retrieved. The implemented search engine is evaluated with 20 students. The results show usefulness and effectiveness on e-learning content searches with a variety of content types, image, document, PDF files, moving picture.
\end{abstract}

Keywords—search engine; API; mashup; Andoride; e-learning content retrieval;

\section{INTRODUCTION}

Mashup technology is defined as search engine with plural different APIs. Mashup has not only the plural APIs, but also the following specific features.

1) it enables classifications of the contents in concern by using web 2.0,

2) it may use API from the different sites,

3) it allows information retrievals from both sides of client and server,

4) it may search contents as an arbitrary structured hybrid contents which is mixed contents formed with the individual contents from the different sites,

5) it enabling to utilize REST, RSS, Atom, etc. which are formed from XML conversions.

There are some Mashup tools, Yahoo! Pipes ${ }^{1}$, Microsoft Popfly ${ }^{2}$, etc. while there are some services using Mashup technology, ChaMap -Enjoy Geo Communication!- ${ }^{3}$, Newsgraphy ${ }^{4}$, Flowser on Amazon ${ }^{5}$

Although Mashup allows content search which is same as portal, Mashup has the aforementioned different features from portal. Therefore, Mashup is possible to create more flexible search engine for any purposes of content retrievals. The search system which is proposed here is that make it possible to control the graph in the $3 \mathrm{D}$ space display with these

\footnotetext{
${ }^{1}$ http://pipes.yahoo.com/pipes/

${ }^{2} \mathrm{http}: / /$ www.microsoft.com/ja-jp/dev/default.aspx

${ }^{3}$ http://chamap.net/

${ }^{4} \mathrm{http}: / /$ newsgraphy.com/

${ }^{5} \mathrm{http}: / / \mathrm{www}$. flowser.com/
}

peculiarity on Android devices. Mashup technology utilized search engine for e-learning content retrievals is proposed. Example of the proposed search engine is implemented with Yahoo!JAPAN Web SearchAPI, Yahoo!JAPAN Image searchAPI, YouTube Data API, and Amazon Product Advertising API. The retrieved results are also merged and linked each other. Therefore, the different types of contents can be referred once an e-learning content is retrieved. The implemented search engine is evaluated with 20 students. The results show usefulness and effectiveness on e-learning content searches with a variety of content types, image, document, PDF files, moving picture.

The following section describes the proposed search engine followed by implementation results and experimental results with 20 students which show usefulness and effectiveness of the proposed content search engine for mobile devices. Then conclusion is described together with some discussions on future investigations.

\section{PROPOSED METHOD AND SYSTEM}

\section{A. Background of the Proposed Search Engine}

Figure 1 shows research background of the proposed search engine.

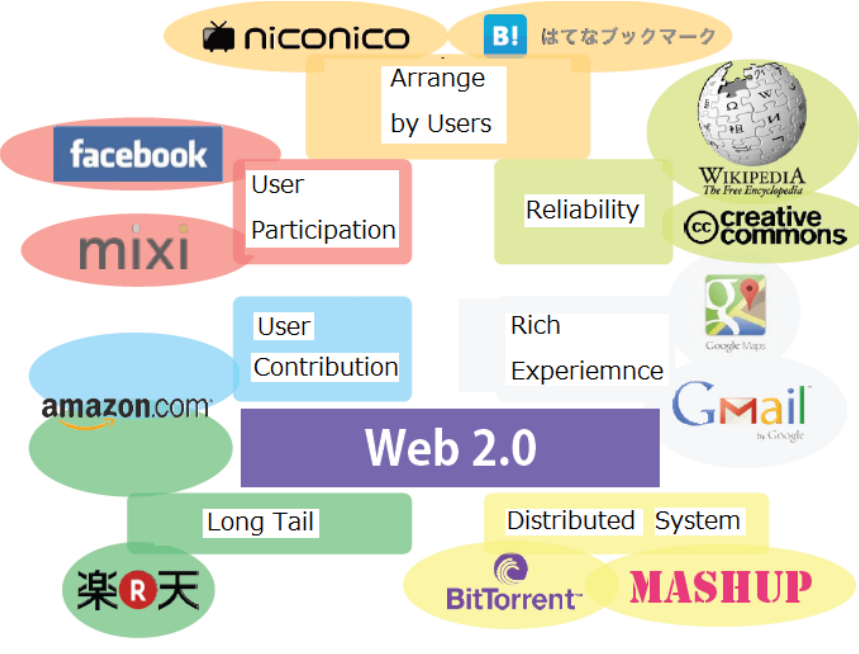

Fig.1. Research background of the proposed mashup based content search engine

There are seven key components, user involvement, user contribution, arranging by users, reliability, rich experiences, 
long tail, and distributed system. Facebook, Mixi, etc. of social network system allows user involvement. Users may contribute to create huge database through amazon.com, for instance. Long tail becomes true with amazon.com, Rakuten, etc, Not only contents but also rearrangement of the contents can be done by users through Hatena Bookmark, Nikoniko, etc. Reliable Q\&A is available through Wikipedia, Yahoo answer, etc. Also rich experiences are obtained through e-mail communications with Gmail, for instance.

These activities can be done based on Web 2.0 technology utilized distributed system. In particular, Mashup technology allows efficient and effective information retrievals for the distributed information providing systems.

\section{B. Content Types and Representation Models for the Content Search}

There are some contents types for search. Namely, document, moving picture, a variety of types of contents, images, etc. Also there are some representation models for the different search content types, helical, star, star-helical, and star slide models. Figure 2 shows an example of star slide model of representation of contents types (Blue star) and the search results (Smile marks). Namely, there are the different content types on the top and there are search results under the content types. With the touch panel function, these content types can be selected (swipe in horizontal direction) and also search results can be selected through swipe in vertical direction.

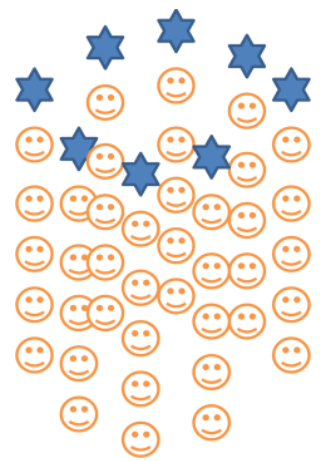

Fig.2. Content type and search result selections by star slide model of representation method

Figure 3 shows five content types as example. Namely, there are Youtube for moving picture, Amazon.com for product, Yahoo for document, Yahoo search for image, and Yahoo for Web searches as shown in Figure 3.

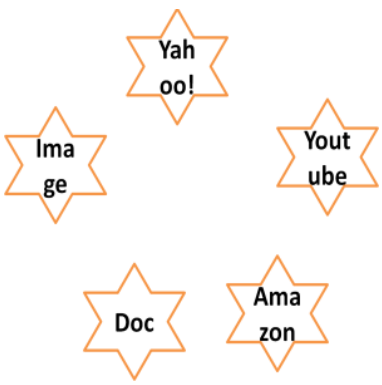

Fig.3. Five different content types are available for search
Under the icon of these five content types, candidate contents are aligned below the icons as search results. In order to show the candidate contents in a 3D representation, the sizes of icons and the candidate contents are changed depending on their locations. Namely, the icons which are located near forward are displayed with relatively large size while those which are located near backward are displayed with comparatively small size as shown in Figure 4.

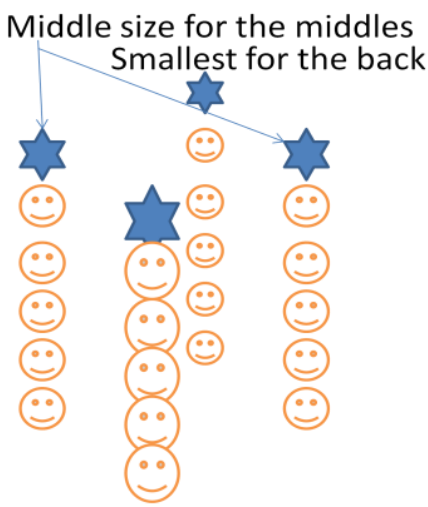

Largest for the front

Fig.4. Icon sizes are changed depending on their locations

Also swipe for candidate URLs in vertical direction and flick for rotation of candidate URLs in horizontal direction are available as shown in Figure 5.

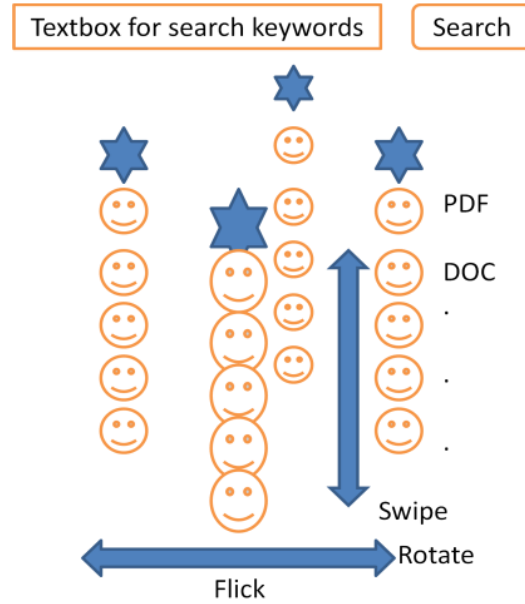

Fig.5. Swipe for candidate URLs in vertical direction and flick for rotation of candidate URLs in horizontal direction

Away3D on Andoroide is used for 3D representation of icons while the APIs which are shown in Figure 6 are used for Mashup. These are aligned in the pentagonal shape as shown in Figure 7. Meanwhile, example of icon movement through swipe for the case of Yahoo search is shown in Figure 8. Candidate of URL icon is selected with swipe (up and down operations). 
Yahoo! Search API

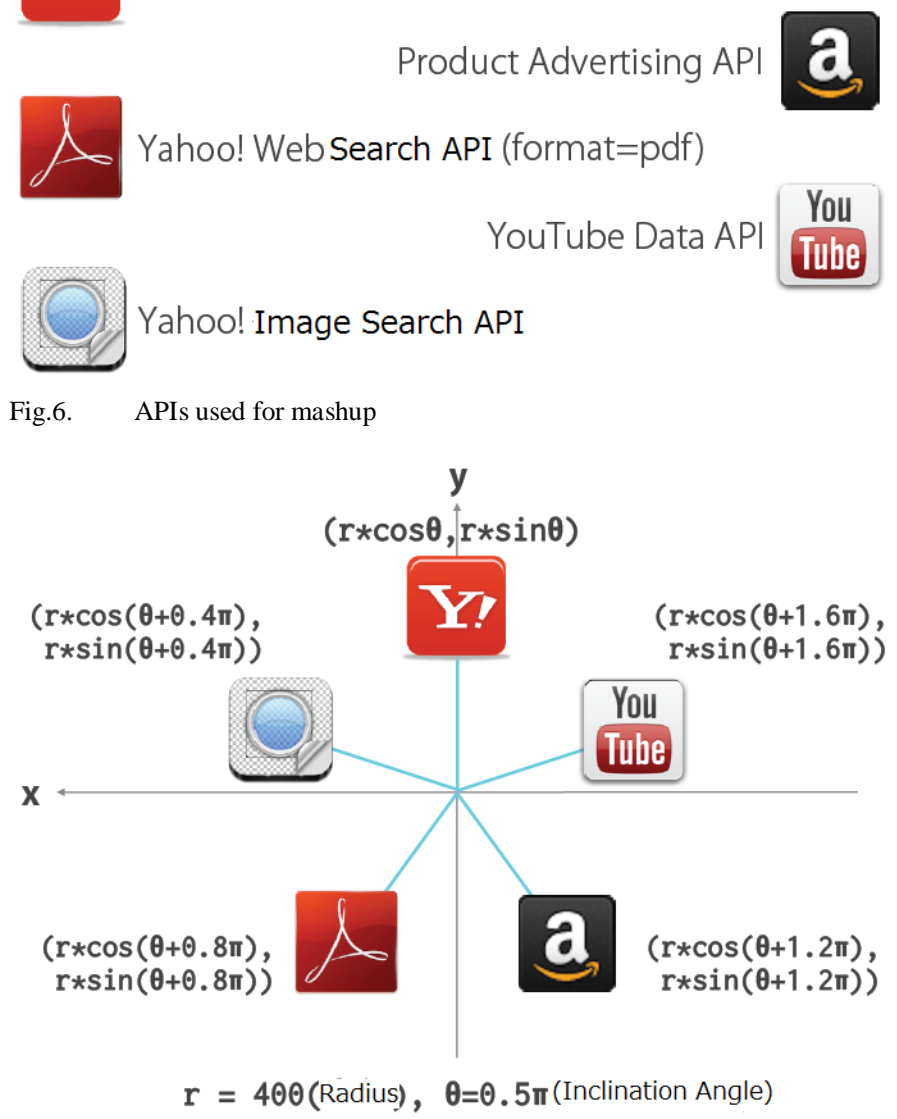

Fig.7. API icons alignment

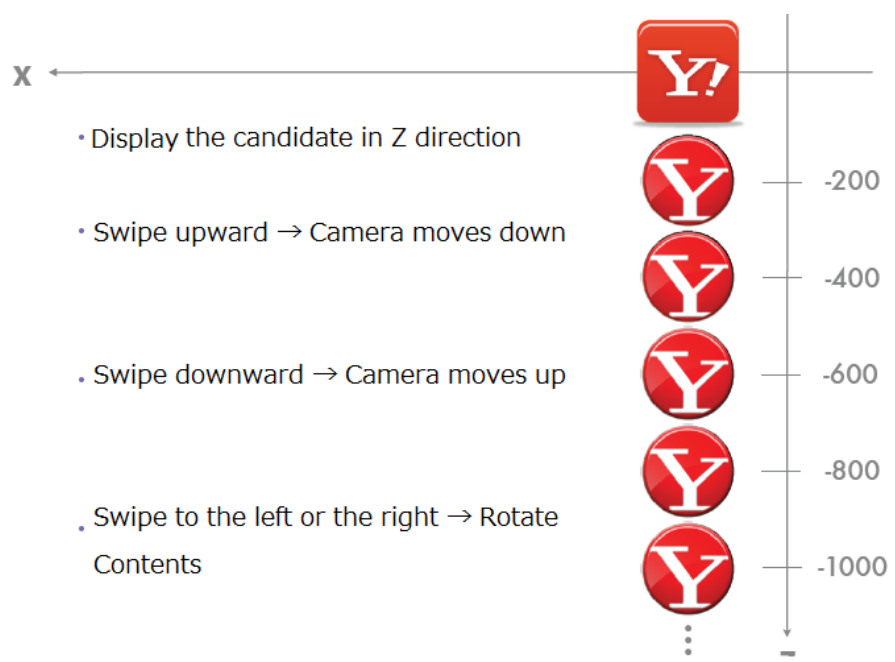

Fig.8. Example of icon movement through swipe for the case of Yahoo search

\section{Examples on Content Search Operations}

The icon of the application software, ELDOXEA: elearning document search engine appears on the screen as shown in Figure 9. ELDOXEA is proposed for e-learning content search [1]-[5].
In this section, example of the search operation with the keyword of "java" is demonstrated as shown in Figure 10. First, users have to key-in their keyword through the dialog box. After the research results appear on the screen, then users have to select the candidate of the search results with swipe and flick operations.

When you click the icon, then Figure 11 (a) of dialog box with search start of radio button appears followed by keyboard screen as shown in Figure 11 (b). When you key-in the keyword, "java" for example, then search result of screen images appear as shown in Figure 12. Examples of swipe and flick operations are shown in Figure 13. Thus users may take a look at research results freely and easily. After that, when user click one icon of the candidate of the search results from the candidates of icons as the search results, the content of the candidate appears as shown in Figure 14.

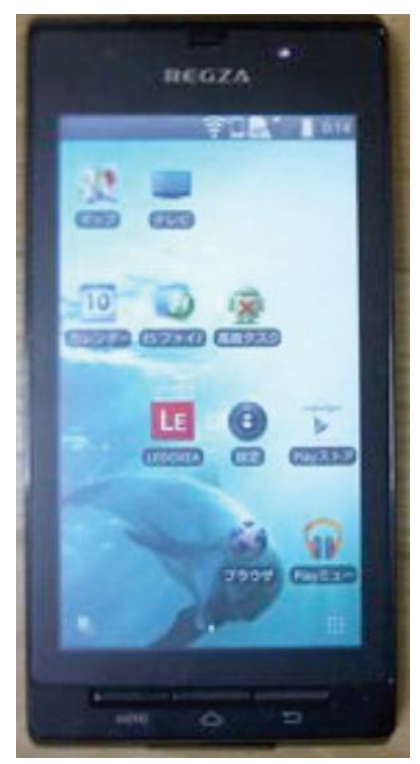

Fig.9. Example of display image of smartphone of which the proposed search engine is implemented

$$
\text { github.com/legnoh/ledoxea }
$$

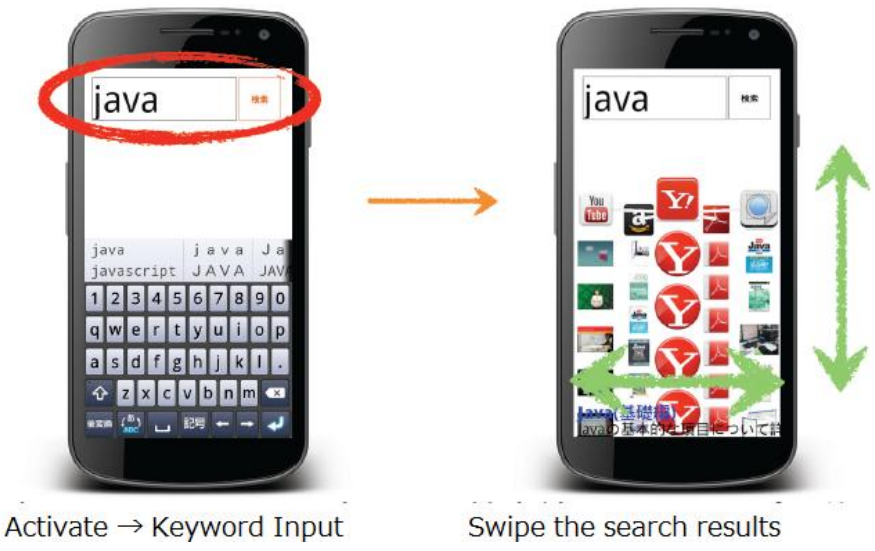

Fig.10. Example operations for search with keyword of "java" 


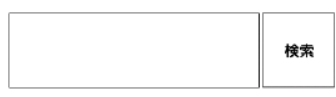

Title

Discription

(a)Search startup screen image

Fig.11. Search startup screen image
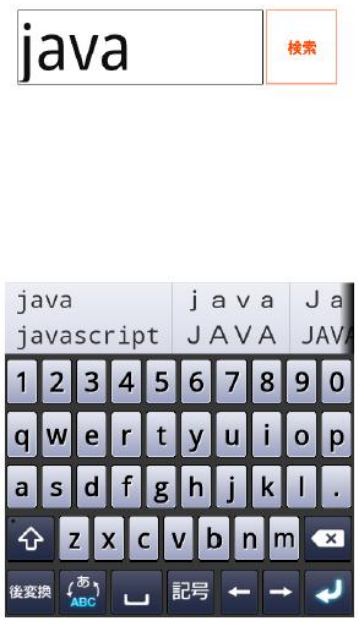

(a)Key-in screen image

Fig.12. Search results of candidates
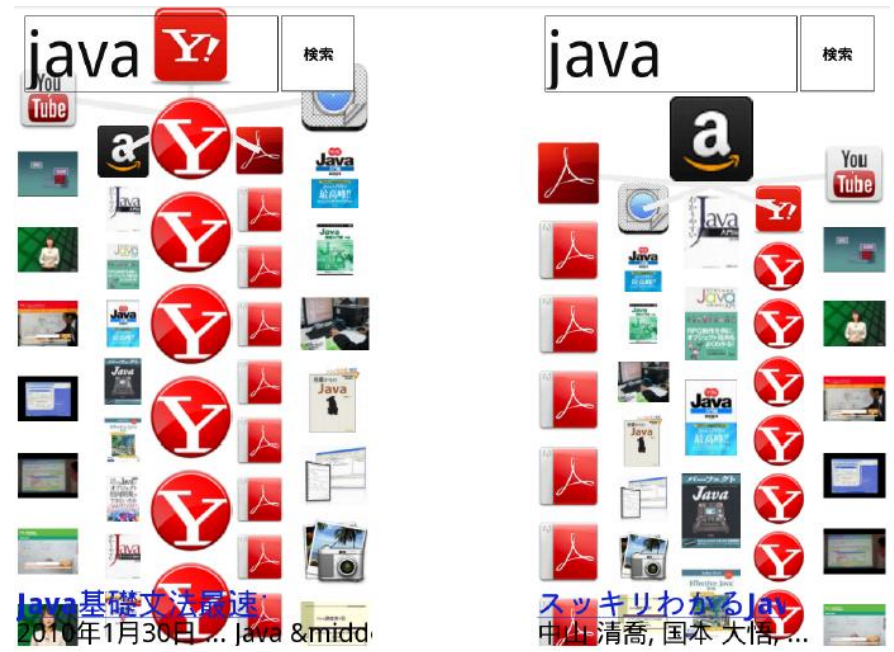

(a)Screen image after vertical swipe (b)Screen image after horizontal flick

Fig.13. Example of search result screen images after swipe and flick operations
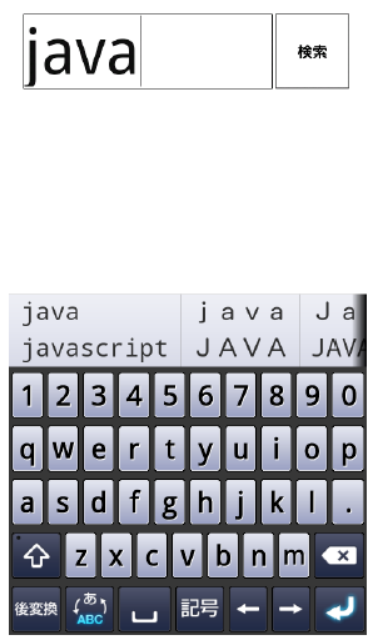

(b)Key-in screen image
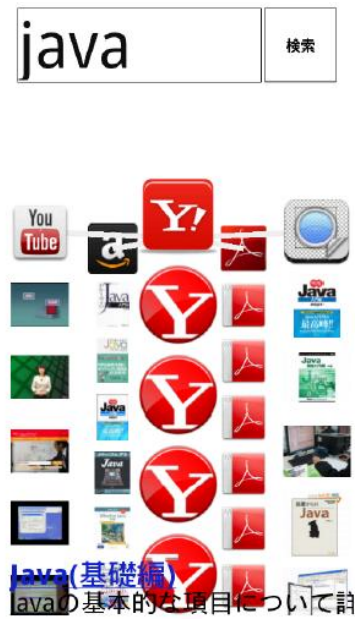

(b)Search results screen image
Fig.15. Example of linked retrieved results (Merged contents of Yahoo search results with the other retrieved contents)

\section{EXPERIMENTS}

20 students in the laboratory of department of information science, Saga University are participated to the experiments using the developed ELDOXEA. Then more than 30 of reports are sent as shown in Figure 16. 

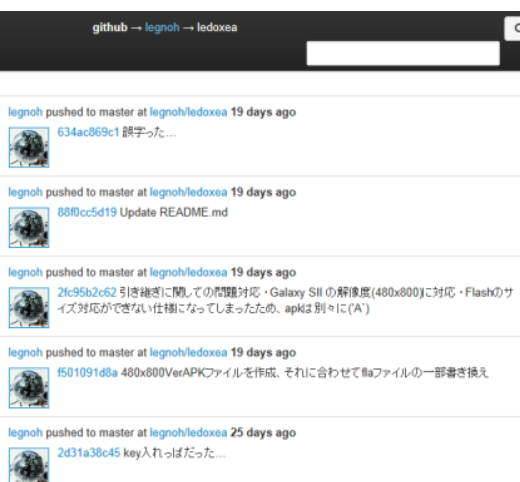

Fig.16. Figure 16 Small portion of the report from the users (20 of students) about ELDOXEA

Although most of those reports are positive, effective search and easy to use, a small number of negative report say that merged contents are not so easy to refer. Link structure has to be clearly shown in the screen.

\section{CONCLUSION}

Mashup based content search engine for mobile devices is proposed. Example of the proposed search engine is implemented with Yahoo!JAPAN Web SearchAPI, Yahoo!JAPAN Image searchAPI, YouTube Data API, and Amazon Product Advertising API. The retrieved results are also merged and linked each other. Therefore, the different types of contents can be referred once an e-learning content is retrieved. The implemented search engine is evaluated with 20 students. The results show usefulness and effectiveness on elearning content searches with a variety of content types, image, document, PDF files, moving picture.

Through experiments with 20 of students who use ELDOXEA for e-learning content search, it is found that the proposed search engine provides e-learning content retrievals comfortably and in a comprehensive manner. On the other hand, there is the report which says that merged contents which are closely related to the retrieved content are not so easy to refer. It should be solved in the future.

\section{ACKNOWLEDGMENT}

The author would like to thank Mr. Ryoma Kai, for his effort to implement search engine application software and to conduct experiments.

\section{REFERENCES}

[1] Kohei Arai,Herman Tolle, Model based content adaptation of conposite e-learning content for delivering to mobile learners, International Journal of Computer Theory and Engineering, 3, 3, 382-387, 2011

[2] Kohei Arai, Herman Tolle, Efficiency improvements of e-learning document search engine for mobile browser, International Journal of Research and Reviews on Computer Science, 2, 6, 1287-1291, 2011.

[3] K.Arai, T.Herman, Efficiency improvement of e-learning document search engine for mobile browser, International Journal of Research and review on Computer Science, 2, 6, 1287-1291, 2012.

[4] K.Arai, T.Herman, E-learning document search method with supplemental keywords derived from keywords in meta-tag and descriptions which are included in the header of the first search result, International Journal of Advanced Computer Science and Applications, 3, 4, 99-104, 2012.

[5] K.Arai, T.Herman, Video searching optimization with supplemental semantic keyword for e-learning video searching, International Journal of Research and Review on Computer Science, 3, 3, 1640-1644, 2012.

\section{AUTHORS PROFILE}

Kohei Arai He received BS, MS and PhD degrees in 1972, 1974 and 1982, respectively. He was with The Institute for Industrial Science and Technology of the University of Tokyo from April 1974 to December 1978 and also was with National Space Development Agency of Japan from January, 1979 to March, 1990. During from 1985 to 1987 , he was with Canada Centre for Remote Sensing as a Post Doctoral Fellow of National Science and Engineering Research Council of Canada. He moved to Saga University as a Professor in Department of Information Science on April 1990. He was a councilor for the Aeronautics and Space related to the Technology Committee of the Ministry of Science and Technology during from 1998 to 2000 . He was a councilor of Saga University for 2002 and 2003. He also was an executive councilor for the Remote Sensing Society of Japan for 2003 to 2005 . He is an Adjunct Professor of University of Arizona, USA since 1998. He also is Vice Chairman of the Commission-A of ICSU/COSPAR since 2008. He wrote 30 books and published 332 journal papers. 